\title{
Machine Learning-based Approach for Prevention of COVID-19 using Steam Vaporizer
}

This paper was downloaded from TechRxiv (https://www.techrxiv.org).

\section{LICENSE}

CC BY 4.0

SUBMISSION DATE / POSTED DATE

20-01-2022 / 26-01-2022

CITATION

Saket, Professor (Dr.) R.K. (2022): Machine Learning-based Approach for Prevention of COVID-19 using Steam Vaporizer. TechRxiv. Preprint. https://doi.org/10.36227/techrxiv.18753377.v1

DOI

10.36227/techrxiv.18753377.v1 


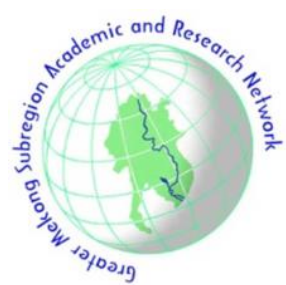

\title{
Machine Learning-based Approach for Prevention of COVID- 19 using Steam Vaporizer
}

\author{
Anand Kumar K.S. ${ }^{1}$, Kumari Sarita ${ }^{2}$, Sachin Kumar $^{2}$, R.K. Saket ${ }^{2}$, and \\ Akshay Swami ${ }^{3}$
}

\section{ART ICLE INFO}

Article history:

Received: 13 June 2021

Revised: 27 August 2021

Accepted: 29 August 2021

\section{Keywords:}

COVID19

SARS

Corona virus

Prevention against COVID19

Steam vaporiser

\begin{abstract}
A B S T R A C T
The present article provides a brief review on Coronavirus Disease-19 (COVID-19) with its prevention using a prototype steam vaporizer developed at home during the lockdown period. The paper discusses the role of Machine Learning (ML) and Artificial Intelligence (AI) for the identification of COVID19. At present, the panic mode and non-availability of anti-drug exponentially increases the COVID-19 cases in India and other parts of the world. This emphasizes humans stay home as a preventing solution to fight against COVID-19. However, staying inside the home is also not safe even when one person is going out for new emergent works. It is also complicated to stay safe from COVID-19 due to a shortage of related preventive measures. Thus, a cheap method of preparing a steam vaporizer is suggested in this paper. Also, its prototype is developed at home with readily available materials. This handheld steam vaporizer has been demonstrated and validated on five people with mild cough and cold symptoms. The survey is carried out using the web page of the COVID 19 survey through the phone. Based on this survey, the person can be informed whether that person is COVID positive or not. The web page for survey is needed to be circulated as much as possible. The tabular and graphically illustrated results show the betterment of this homemade steam vaporizer in mitigating the $\mathrm{CoV}$ like viruses.
\end{abstract}

\section{INTRODUCTION}

The dispersion of COVID-19 and its transmission has been studied from the literature, and it is observed that the high temperature and humidity affect the lifetime of COVID-19 viruses (if subjected for a certain period of time). The authors in [1] have focused on the effect of high temperature and high humidity in mitigating the COVID viruses. The paper focuses on a brief review, including CoV's spreading and its prevention using a developed steam vaporizer. To study the developed precautions and treatments, it is necessary to understand the stability of the virus in varying temperature and humidity conditions. This will definitely help in the analysis of the rate of transmission of the virus. The main reason of transmission of COV virus is the droplets of the respiration and close contacts [2-6]. From a survey [1], the authors have observed that the dried virus retains its viability for over five days on smooth surfaces at the temperature range of $22-25^{\circ} \mathrm{C}$ and relative humidity of $40-50 \%$. It is the environment of a typical air-conditioner based area. The virus's viability is rapidly lost to a great extent at higher temperatures and higher relative humidity of range
$38 \$^{\wedge} 0 \mathrm{C} \$$ and greater than $95 \%$, respectively. The authors [1] reported that viability of SARS CoV was lost after temperature treatment at $56^{\circ} \mathrm{C}$ for 15 minutes [7]. Authors [8-10] have summarized the persistence of human viruses like SARS, MERS, or CoV on inanimate surfaces. They have explained that these viruses can persist on metal, glass, or plastic for up to 9 days and also can be inactivated by treating the material with $62-71 \%$ ethanol, $0.5 \%$ hydrogen peroxide, or $0.1 \%$ sodium hypochlorite within one minute. The authors have summarized the inactivation of coronaviruses by different types of biodal agents in suspension tests and carrier tests.

Further, it becomes essential to study the behavior of the virus with its symptoms. Also, the number of infected, deceased, and recovered persons are required to be analyzed through any ML or/and AI technique/s [11]. It is because of fast diagnosis and treatment solutions for the protection of humanity. The authors in [12] have provided insight into AI and big data to identify the applications that aim to reduce the effect of COVID-19. Also, some deep learning methods have been described for physicians and researchers to accelerate the diagnosis and treatment

\footnotetext{
${ }^{I}$ Division of Electrical Engineering, CSIR-National Aerospace Laboratories,Bangalore, Karnataka, India.

${ }^{2}$ Department of Electrical Engineering, Indian Institute of Technology (Banaras Hindu University),Varanasi, Uttar Pradesh, 221005 India.

${ }^{3}$ Bengal Homeopathic Medical College \& Hospital, Ismile, Asansol (WB), India.

*Corresponding author: R.K. Saket; Email: rksaket.eee@iitbhu.ac.in.
} 
process [13]. Simultaneously, the data provided should be authentic to avoid any false reports and rumors during this pandemic situation. Some researchers have given their opinion on the implementation of the internet of things, AI, and other methods for COVID-19 outbreak impact mitigation [14, 25-27].

It is observed from the above literature survey that the actual treatment is still not confirmed for COVID-19, and the research work is still on the process for the treatment of this pandemic [15-16]. In most of the research papers, researchers have suggested hot water and hot air as a preventive solution against COVID. The authors of this article have observed that steam therapy is extremely useful in minimizing the menace of $\mathrm{CoV}$ as the temperature of the steam is near to the boiling temperature, which goes through the upper respiratory tract and reaches the alveoli, which moisten and thereby helps in increasing the $\mathrm{O}_{2}$ saturation. This paper proposes a simple method for taking steam vapor using a steam vaporizer, which can be prepared at home during the lock-down period. It is expected that this paper provides researchers and communities with new insights into the ways machine learning and big data improve the COVID-19 situation and creates further research opportunities in stopping the COVID-19 outbreak.

The rest of the article has been organized as follows. The Epidemiology is briefly described in Section 2. The COVID-19 symptoms and its precautions are also described in Section 2. With its experimental graphical results, the proposed method is discussed in Section 3. Finally, the conclusion and scope for future work are mentioned in Section 4.

\section{PROPOSED METHODOLOGY}

\subsection{Epidemiology}

India is a highly populated country and stands 2nd in the world. So, it becomes very crucial to study and analyze the dispersal, structure, and determinants of health and disease conditions in defined populations [17-19]. It was observed that many pneumonia cases from Wuhan city of PRC were investigated in November 2019-December2020. The outcome of in-depth exploration describes the source of COVID-19 is from Huanan Seafood Market in Wuhan city. The first case of the COVID-19 pandemic was investigated with undescribed pneumonia, and 27 more such cases were officially announced in December 2019 by the government of PRC [20-22]. The performed Etiological investigations have shown that an infection is likely transmitted from any animal to human. Then, it is announced that $\mathrm{CoV}$ has come from wild bats. This $\mathrm{CoV}$ belongs to Group 2 of beta-CoV that contains SARS-CoV. Despite the same beta-CoV subgroup, SARS-CoV is similar only $70 \%$ at the genome level. On the other hand, SARS-CoV 2 is a novel group that shows genetic differences from SARS-CoV.

It is observed that this outbreak has taken place during the Spring Festival in PRC. Nearly 3 billion people travel countrywide during this festival [17] as these situations are favorable for the transmission of this disease. Thus, the first case of India was confirmed on 30th January 2020 from the state of Kerala. The first case of India is due to the person who traveled from PRC. The large-scale travel from the PRC to other parts of the world has also created a favorable condition for the dispersion of COVID-19.

\subsection{Covid-19 symptoms and its treatment through precaution}

The primary symptoms of COVID-19 are similar to any other viral infection. Some of the symptoms are as follows [23-24].

(i) Sneezing, (ii) runny nose, (iii) cough, (iv) diarrhea, (v) fever and pain, (vi) sore throat, and (vii) exacerbated asthma.

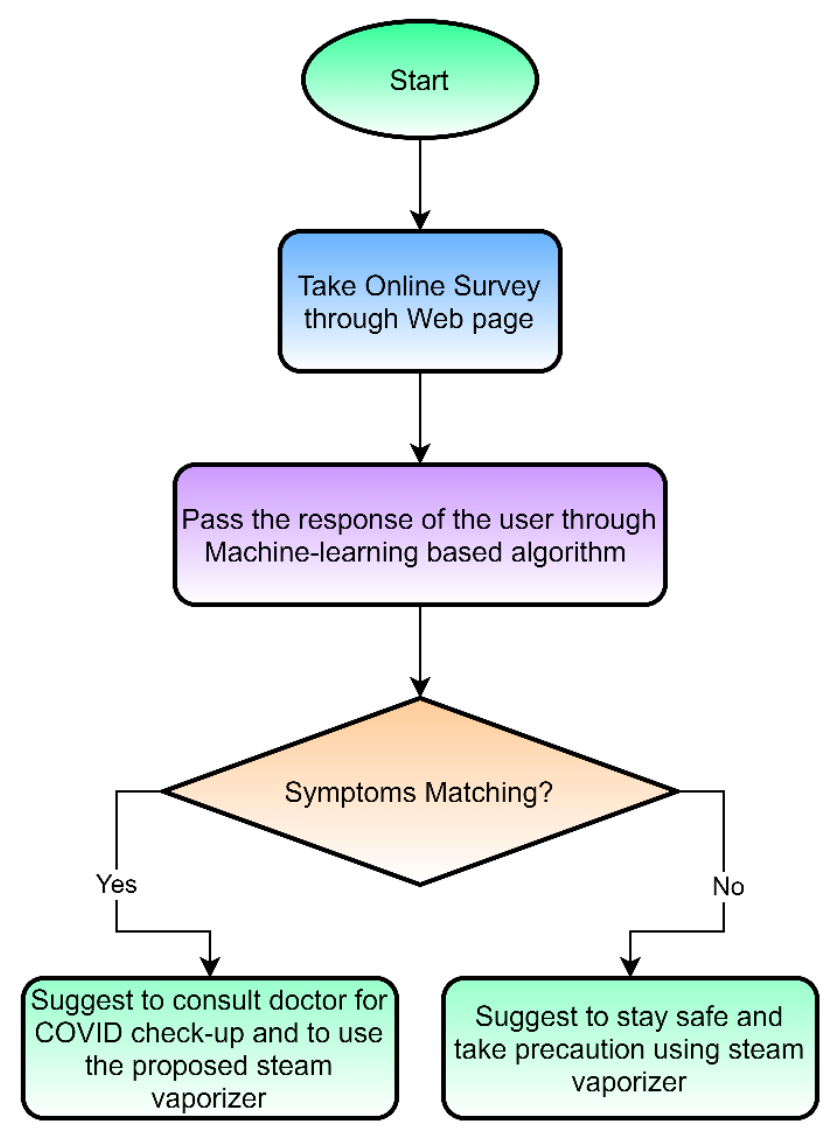

Fig. 1: Flow chart of the proposed methodology.

Flu or cold-like symptoms are easily curable in 3-5 days, but COVID-19 takes up to 2-3 weeks for cure or may lead to death due to organ failure or some other problems. However, these symptoms are not fixed and vary from person to person. The researchers and doctors are still unable to discover the anti-drug of COVID-19, but few suggestions with precautions are given as follows for the people to prevent or cure this disease.

(i) Take rest to avoid exertion, (ii) drinking enough warm 
water, (iii) keep yourself away from polluted areas, (iv) take prescribed medicine for fever and pain, (v) use a clean humidifier or steam vaporizer, (vi) consult a doctor at earliest, (vii) standard recommendations to prevent infection spread, (viii) avoid smoking and (ix) wash hands regularly, use a face mask and maintain social distancing.

Thus, the present article is further focused on the prototype development of steam vaporizer during the lock-down period using readily available materials at home. The flow chart of the proposed methodology is shown in Figure 1.

\section{RESULTS AND DISCUSSION}

\section{COVID-19 survey through phone}

The survey is taken place through phone using a web survey form including the information regarding place, age, contact number, and the symptoms (if any). The web survey form is made in two parts as shown in Figure 2 and Figure 3.

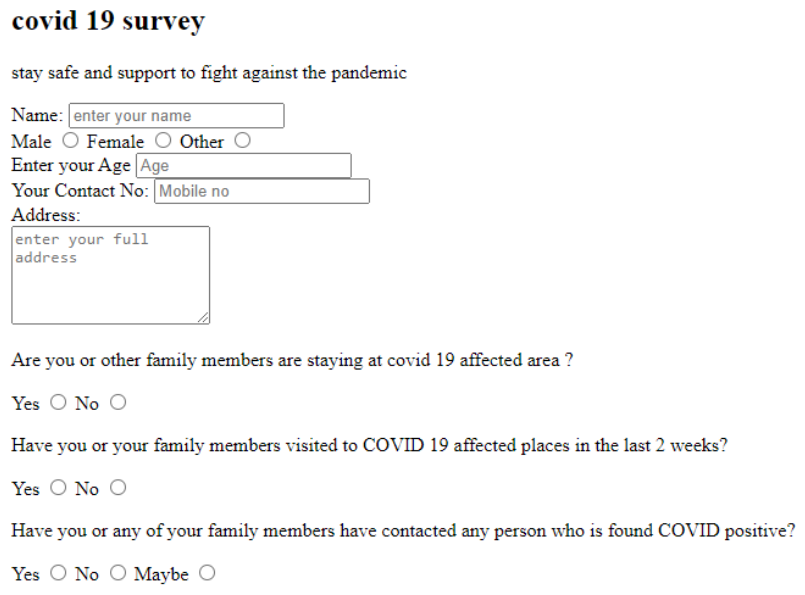

Fig. 2: First part of survey form for COVID-19.

covid 19 symptoms

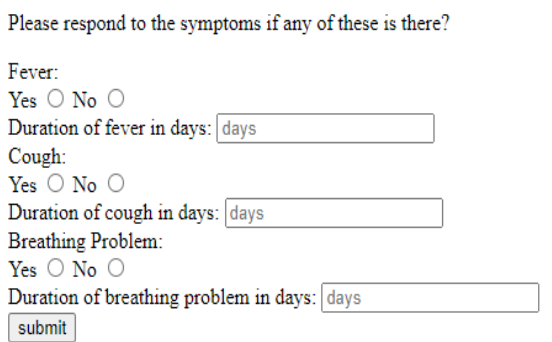

Fig. 3: Second part of survey form for COVID-19.

\section{Machine learning approach}

The proposed algorithm can help identify possible cases, and it can be used to collect information to send an alert to the nearest health center and the person who has submitted the symptoms with appropriate suggestions. This way, people can be sure if they are safe or not in a particular living place. If a person does not have any symptoms or signs related to the COVID-19, then the proposed algorithm can send a message to the person to inform that there is no risk of COVID-19 at the current time.

With the help of data science and machine learning, diagnosis and treatment can be reliable and fast. The data collected from the web survey, as discussed in the previous Section 4.1 are used to identify the problem. For this, different possible categories of diseases are collected, and the corresponding symptoms are also grouped for the learning of the diagnosis system. The symptoms and travel/contact history information are considered as the indicators to predict the predictor and identify the disease. The indicators, including symptoms and travel history, are as given in Table 1.

Table 1. Experimental data of five-person after using developed steam vaporizer prototype

\begin{tabular}{|c|l|}
\hline Name & \multicolumn{1}{|c|}{ Description } \\
\hline$T_{1}$ & $\begin{array}{l}\text { The person or any of family member are } \\
\text { staying/working at COVID-19 affected area }\end{array}$ \\
\hline$T_{2}$ & $\begin{array}{l}\text { The person or any of family member have visited } \\
\text { COVID-19 affected area in last 14 days }\end{array}$ \\
\hline$T_{3}$ & $\begin{array}{l}\text { The person or any of family members have } \\
\text { contacted any person who is COVID positive. }\end{array}$ \\
\hline$S_{1}$ & Having fever \\
\hline$D_{s}$ & Duration of fever \\
\hline$S_{2}$ & Having cough \\
\hline$D_{\mathrm{s} 2}$ & Duration of cough \\
\hline$S_{3}$ & Breathing problem \\
\hline$D_{\mathrm{s} 3}$ & Duration of breathing problem \\
\hline
\end{tabular}

If $C_{1}$ is the identifier function including the indicators $S_{1}$, $S_{2}, D_{s 1}, D_{s 2}$ and $C_{2}$ is the class of disease indicators $S_{1}, S_{2}$, $S_{3}, D_{s 1}, D_{s 2}$, and $D_{s 3}$. Any person can have one, more than one or none of these symptoms or indications. Based on the positive and negative results of the symptoms, the predictor function is formulated as follows. The predictor $\mathrm{P}_{1}$ for the $\mathrm{C}_{1}$ class of indicators can be any matching element from the group of possible matches of indicators.

$$
P_{1}=\left\{\begin{array}{c}
(1,1,1,1) \\
(0,1,1,1) \\
(1,1,0,1) \\
(1,0,1,1) \\
(1,1,1,0) \\
\vdots \\
(0,1,1,0)
\end{array}\right\}
$$

Similarly, the predictor $\mathrm{P}_{2}$ also consists of a group of symptoms given as follows. 


$$
P_{2}=\left\{\begin{array}{c}
(0,0,1,1) \\
(0,1,0,1) \\
(1,0,0,1) \\
(1,0,1,0) \\
(1,1,0,0) \\
\vdots \\
(0,1,0,0)
\end{array}\right\}
$$

Based on the class of predictor matching with a particular set of symptoms and corresponding particular disease, the person can be informed about the health status and chances of becoming COVID positive. Thus, the role of machine learning and artificial intelligence comes here. The machine learning-based model can be trained for a particular disease with its different possible symptoms. With the help of artificial intelligence, the number of positive cases in any particular area can be predicted. The Support Vector Machine (SVM)-based machine learning technique has been used in this paper.

Based on the proposed web page survey, a few persons are collected and categorized into different predictors for diagnosis. It is observed that many people are suffering from cold and fever. To control these and avoid COVID attacks, the people were suggested to follow the government's guidelines against COVID. For killing the virus-like SARS and COVID, the suggested steam vaporizer is discussed in this Section.

\section{Steam vaporizer at Home}

The materials required for this steam vaporizer include a thermos and water bottleneck. This methodology of preparing a steam vaporizer at home is straightforward. The materials required for this are shown in Figure 4(a). The $500 \mathrm{~mL}$ of water is taken and boiled; then, this boiled water is filled into a thermos. The water bottleneck is placed in the thermos' opened mouth portion, as shown in Figure 4(b). To make this steam vaporizer more comfortable, the arrangement shown in Figure 4(c) is made.

It is observed that the hot water in the thermos starts evaporating and allowing heat and steam to penetrate the nose and sinuses. While taking this hot water vapor, it must take slow breaths with mouth closed for at least five minutes. This will definitely raise the temperature of the sinuses to $56^{\circ} \mathrm{C}$. The repetition of this process after one or two hours mitigates the coronaviruses of the human. If there are no such symptoms, then doing this process twice or thrice in a day is sufficient to prevent these viruses from humans.

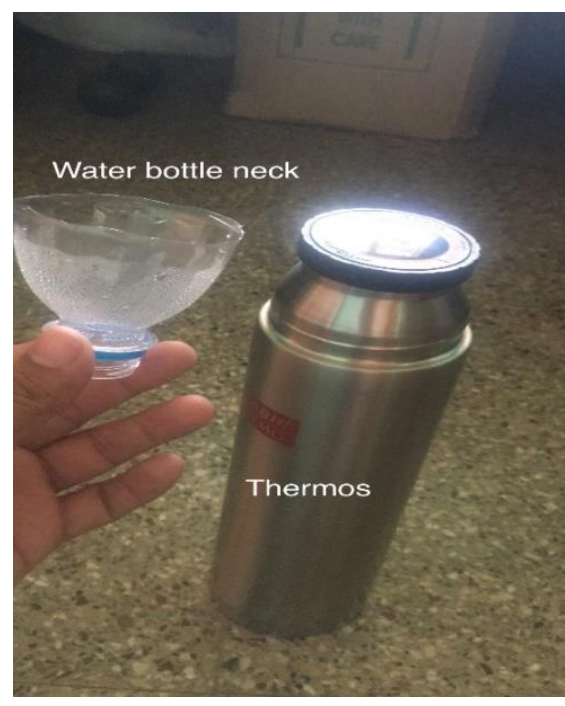

(a) Materials required

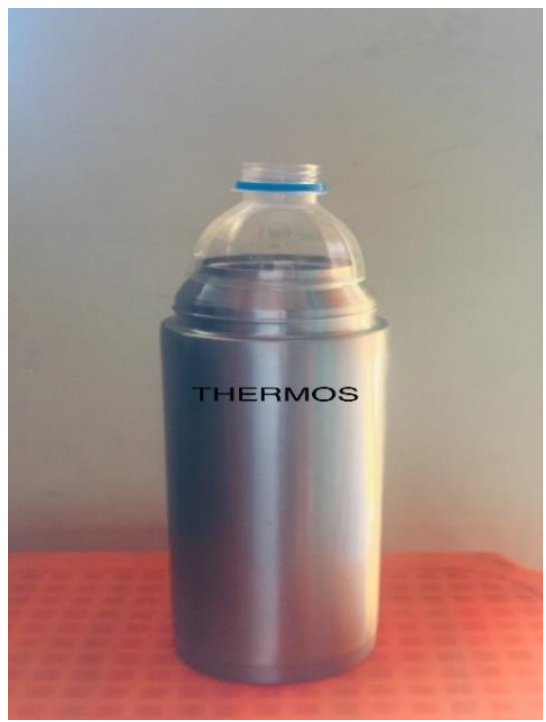

(b) Prepared steam vaporiser

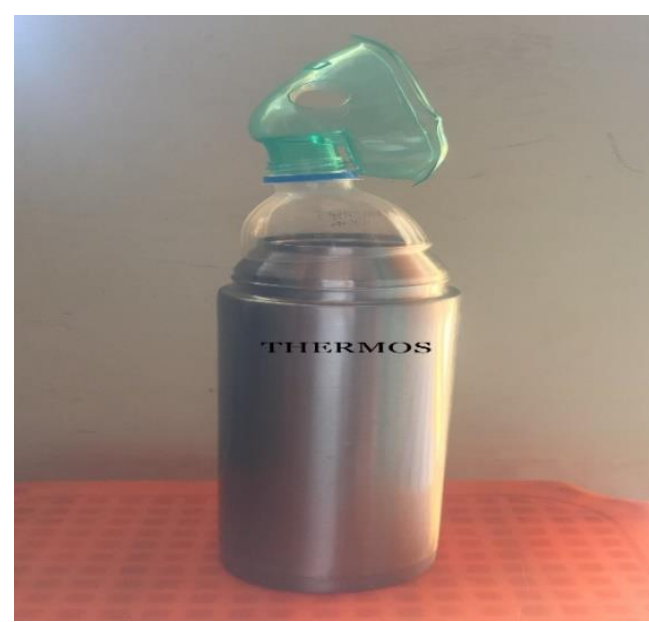

(c) Nebulizer arrangement

Fig. 4: Prototype development of steam vaporiser. Table 2. Experimental data of five people after using 
developed steam vaporizer prototype

\begin{tabular}{|c|c|c|c|c|}
\hline S. No. & Days & Cough & Sneezing & $\begin{array}{c}\text { Body } \\
\text { Temperature } \\
\left({ }^{0} \mathrm{~F}\right)\end{array}$ \\
\hline \multirow[t]{5}{*}{$1^{\text {st }}$ Person } & 1 & 5 & 20 & 101.6 \\
\hline & 2 & 15 & 10 & 101.2 \\
\hline & 3 & 18 & 10 & 100 \\
\hline & 4 & 10 & 6 & 98 \\
\hline & 5 & 4 & 3 & 98.4 \\
\hline \multirow[t]{5}{*}{$2^{\text {nd }}$ Person } & 1 & 6 & 22 & 100.6 \\
\hline & 2 & 14 & 13 & 100 \\
\hline & 3 & 19 & 9 & 99.6 \\
\hline & 4 & 8 & 5 & 99 \\
\hline & 5 & 5 & 1 & 98.6 \\
\hline \multirow[t]{5}{*}{$3^{\text {rd }}$ Person } & 1 & 5 & 22 & 100 \\
\hline & 2 & 14 & 14 & 100.4 \\
\hline & 3 & 18 & 10 & 99.8 \\
\hline & 4 & 9 & 5 & 98.8 \\
\hline & 5 & 6 & 3 & 98 \\
\hline \multirow[t]{5}{*}{$4^{\text {th }}$ Person } & 1 & 11 & 28 & 102.2 \\
\hline & 2 & 20 & 19 & 101.6 \\
\hline & 3 & 23 & 11 & 100 \\
\hline & 4 & 15 & 10 & 100 \\
\hline & 5 & 12 & 10 & 99 \\
\hline \multirow[t]{5}{*}{$5^{\text {th }}$ Person } & 1 & 6 & 18 & 99.6 \\
\hline & 2 & 10 & 12 & 99 \\
\hline & 3 & 10 & 6 & 99 \\
\hline & 4 & 8 & 3 & 98.6 \\
\hline & 5 & 5 & 2 & 98 \\
\hline
\end{tabular}

\begin{tabular}{|ccc|}
\hline -Body Temperature & -Cough & - Sneezing \\
\hline - Body Temperature & - Cough & - Sneezing \\
\hline -Body Temperature & - Cough & - Sneezing \\
\hline - Body Temperature & - Cough & - Sneezing \\
\hline - Body Temperature & - Cough & - Sneezing \\
\hline
\end{tabular}

Fig. 5: The experimental test results of three symptoms of all five persons.

The developed prototype of steam vaporizer is tested on five persons having mild symptoms of cough, cold, and fever for five days as given in Table 2 and illustrated graphically in Figure 5. The graphs are shown in Figure 5 describe the variation of body temperature, and cough and sneezing for five days in each person. It is illustrated that the day-wise recorded temperatures of all five persons are decreasing by inhaling hot air through a developed steam vaporizer. Simultaneously, the use of this vaporizer shows that the cough per day increases for the first three days then controlled at the end of day 5 . The additional benefit of a developed steam vaporizer is observed that the sneezing per day is high on day 1 , and then its frequency decreases to normal at the end of day 5 .

\section{CONCLUSION}

The role of machine learning and artificial intelligence made it possible to identify the COVID 19 positive cases. The particular person is given information regarding the same. For preventing the spread of COVID-19, some precautionary measure has been discussed. Prevention is always better than cure, especially to fight against COVID19. The researchers are still in progress on exploring the correct treatment and drug for COVID-19. It is observed that there is an unavailability of the vaccine for this virus. So, some primary measures to prevent this virus are suggested. The suggestions include maximizing the stay at home, regular washing of hands, frequent use of sanitizer, and steam vaporizer. This article has given a brief review of COVID-19 and proposed a steam vaporizer that is simple and easy to prepare at home during the lock-down period. It helps in preventing the COVID-19 up to an extent as proven experimentally. The five persons with a mild cough, sneezing, and fever symptoms are tested with a developed steam vaporizer for five days. These persons' test results are too favorable that at the end of day five, as all three symptoms are mitigated by inhaling hot vapor through the developed steam vaporizer. This act of preventing COVID-19 could mitigate the viruses of the nose, face, and hands using a steam vaporizer. In the places where the stock of sanitizer and vaporizer is not readily available and out of reach, this developed steam vaporizer could be a better alternative. The proposed steam vaporizer has been validated and found effective treatment in mitigating the CoV. Thus, inhaling hot steam is an effective solution developed and suggested to prevent COVID-19. Further, research can be done on the commercialization of vaporizers and other methods to mitigate this pandemic disease.

\section{ACKNOWLEDGMENT}

The authors are deeply appreciating the resources for their efforts from the Division of Electrical Engineering, CSIR-National Aerospace Laboratories, Bangalore, Karnataka, India and Indian Technology Institute (Banaras Hindu University), Varanasi, Uttar Pradesh, India.

\section{REFERENCES}

[1] K.-H. Chan, J. M. Peiris, S. Lam, L. L. Poon, K.-Y. Yuen, W. H. Seto, The effects of temperature and relative humidity on the viability of the sars coronavirus, Advances in virology 2011 . 
[2] J. Tan, L. Mu, J. Huang, S. Yu, B. Chen, J. Yin, An initial investigation of the association between the sars outbreak and weather: with the view of the environmental temperature and its variation, Journal of Epidemiology \& Community Health 59 (3) (2005) 186-192.

[3] J. Yuan, H. Yun, W. Lan, W. Wang, S. G. Sullivan, S. Jia, A. H. Bittles, A climatologic investigation of the sars-cov outbreak in beijing, china, American journal of infection control 34 (4) (2006) 234-236.

[4] Q.-C. Cai, J. Lu, Q.-F. Xu, Q. Guo, D.-Z. Xu, Q-W. Sum, H. Yang, G.-M. Zhao, Q.-W. Jiang, Influence of meteorological factors and air pollution on the outbreak of severe acute respiratory syndrome, Public Health 121 (4) (2007) 258-265.

[5] W. Seto, D. Tsang, R. Yung, T. Ching, T. Ng, M. Ho, L. Ho, J. Peiris, A. of Expert SARS group of Hospital Authority, et al., Effectiveness of precautions against droplets and contact in prevention of nosocomial transmission of severe acute respiratory syndrome (sars). The Lancet 361 (9368) (2003) 1519-1520.

[6] M. A. Shereen, S. Khan, A. Kazmi, N. Bashir, R. Siddique, Covid-19 infection: Origin, transmission, and characteristics of human coronaviruses, Journal of Advanced Research.

[7] M. E. Darnell, K. Subbarao, S. M. Feinstone, D. R. Taylor, Inactivation of the coronavirus that induces severe acute respiratory syndrome, sars-cov, Journal of virological methods 121 (1) (2004) 85-91.

[8] G. Kampf, D. Todt, S. Pfaender, E. Steinmann, Persistence of coronaviruses on inanimate surfaces and their inactivation with biocidal agents, Journal of Hospital Infection 104 (3) (2020) 246-251.

[9] G. Kampf, Potential role of inanimate surfaces for the spread of coronaviruses and their inactivation with disinfectant agents, Infection Prevention in Practice 2 (2) (2020) 100044.

[10] C.-C. Lai, T.-P. Shih, W.-C. Ko, H.-J. Tang, P-R. Hsuch, Severe acute respiratory syndrome coronavirus 2 (sars-cov2) and corona virus disease-2019 (covid-19): the epidemic and the challenges, International journal of antimicrobial agents (2020) 105924.

[11] F. Rustam, A. A. Reshi, A. Mehmood, S. Ullah, B. On, W. Aslam, G. S. Choi, Covid-19 future forecasting using supervised machine learning models, IEEE Access.

[12] Q. Pham, D. C. Nguyen, T. Huynh-The, W. Hwang, P. N. Pathirana, Artificial intelligence (ai) and big data for coronavirus (covid-19) pandemic: A survey on the state-ofthe-arts, IEEE Access 8 (2020) 130820-130839.

[13] M. Jamshidi, A. Lalbakhsh, J. Talla, Z. Peroutka, F. Hadjilooci, P. Lalbakhsh, M. Jamshidi, L. La Spada, M. Mirmozafari, M. Dehghani, et al., Artificial intelligence and covid-19: deep learning approaches for diagnosis and treatment, IEEE Access 8 (2020) 109581-109595.

[14] V. Chamola, V. Hassija, V. Gupta, M. Guizani, A comprehensive review of the covid-19 pandemic and the role of iot, drones, ai, blockchain, and $5 \mathrm{~g}$ in managing its impact, IEEE Access 8 (2020) 90225-90265.
[15] I. Thevarajan, T. H. Nguyen, M. Koutsakos, J. Druce, L. Caly, C. E. van de Sandt, X. Jia, S. Nicholson, M. Catton, B. Cowie, et al., Breadth of concomitant immune responses prior to patient recovery: a case report of non-severe covid19, Nature medicine 26 (4) (2020) 453455.

[16] C. S. G. of the International, et al., The species severe acute respiratory syndrome-related coronavirus: classifying 2019ncov and naming it sars-cov-2, Nature Microbiology 5 (4) (2020) 536.

[17] A. K. Bhardwaj, R. K. Chandra, A review on coronavirus (covid-19) communicable disease in relation to human health perspective, EPIDEMIOLOGY AND TRANSMISSION OF INFECTIOUS DISEASES (2020) 17-37.

[18] S. P. Adhikari, S. Meng, Y.-J. Wu, Y.-P. Mao, R.-X. Ye, Q.Z. Wang, C. Sun, S. Sylvia, S. Rozelle, H. Raat, et al., Epidemiology, causes, clinical manifestation and diagnosis, prevention and control of coronavirus disease (covid-19) during the early outbreak period: a scoping review, Infectious diseases of poverty 9 (1) (2020) 1-12.

[19] A. J. Jinia, N. B. Sunbul, C. A. Meert, C. A. Miller, S. D. Clarke, K. J. Kearfott, M. M. Matuszak, S. A. Pozzi, Review of sterilization techniques for medical and personal protective equipment contaminated with sars-cov-2, IEEE Access 8 (2020) 111347-111354.

[20] R. J. Lin, T. H. Lee, D. C. B. Lye, From sars to covid-19: the singapore journey, The Medical Journal of Australia 6.

[21] K. G. Andersen, A. Rambaut, W. 1. Lipkin, E. C. Holmes, R. F. Garry, The proximal origin of sars-cov-2, Nature medicine 26 (4) (2020) 450-452.

[22] B. Xu, B. Gutierrez, S. Mekaru, K. Sewalk, L. Goodwin, A. Loskill, E. L. Cohn, Y. Hswen, S. C. Hill, M. M. Cobo, et al., Epidemiological data from the covid-19 outbreak, realtime case information, Scientific data 7 (1) (2020) 1-6.

[23] M. Letko, A. Marzi, V. Munster, Functional assessment of cell entry and receptor usage for sars-cov-2 and other lineage b betacoronaviruses, Nature microbiology 5 (4) (2020) 562569.

[24] K. Michael, R. Abbas, R. A. Calvo, G. Roussos, E. Scornavacca, S. F. Wamba, Manufacturing consent: The modern pandemic of technosolutionism, IEEE Transactions on Technology and Society 1 (2) (2020) 68-72.

[25] Panday, Aishwarza, Muhammad Ashad Kabir, and Nihad Karim Chowdhury. "A Survey of Machine Learning Techniques for Detecting and Diagnosing COVID-19 from Imaging." arXiv preprint arXiv:2108.04344 (2021).

[26] Kumar, Swarn Avinash, Harsh Kumar, Vishal Dutt, and Pooja Dixit. "The Role of Machine Learning in COVID-19 in Medical Domain: A Survey." Journal on Recent Innovation in Cloud Computing, Virtualization \& Web Applications [ISSN: 2581-544X (online)] 4, no. 1 (2020).

[27] El-Rashidy, Nora, Samir Abdelrazik, Tamer Abuhmed, Eslam Amer, Farman Ali, Jong-Wan Hu, and Shaker ElSappagh. "Comprehensive Survey of Using Machine Learning in the COVID-19 Pandemic." Diagnostics 11, no. 7 (2021): 1155. 